\title{
insonorización en el aeropuerto de HAMBURGO
}

H. KONRAD HAVEMANN, Dr. ingeniero

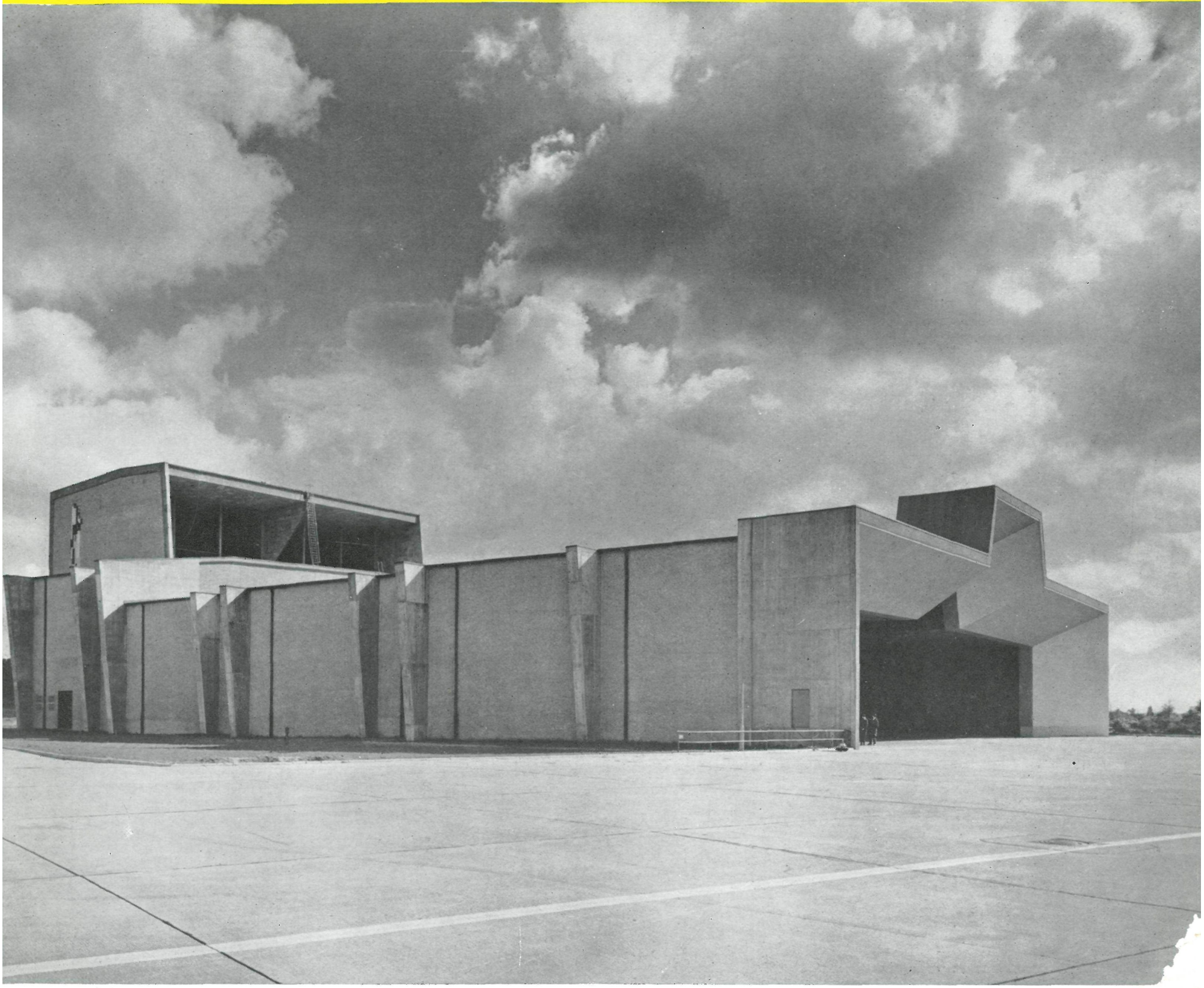

$545-30$

simopsis

Los aeropuertos, particularmente los más antiguos, presentan actualmente el problema del ruido, ya que cuando se construyeron se hallaban enclavados en zonas apenas habitadas. La expansión y diario crecimiento del radio urbano de las grandes ciudades lleva los barrios residenciales a las inmediaciones de los aeropuertos y, con ello, aparece el problema de la insonorización, pues deben evitarse, o por lo menos atenuar, las molestias que los ruidos excesivos crean en las zonas de habitación.

Para dar solución a este estado de cosas se ha emprendido, en Hamburgo, la construcción de un hangar de insonorizaciór de los ruidos que los aparatos modernos hacen al poner sus motores en marcha. El tiempo de calentamiento de motores, espera, etc., lo pasará el aparato en marcha en el interior de este hangar.

Los ensayos realizados del hangar en servicio han dado resultados satisfactorios.

La estructura general es de hormigón pretensado, con fuertes pórticos transversales. Suspendido del techo se ha dispuesto un sistema de placas acústicas que servirán para amortiguar los ruidos.

El edificio presenta una forma original, recia y de plástica característica. 


\section{Exposición genemerent}

El aeropuerto de Hamburgo, Alemania, construido hace cincuenta años, no constituye un caso particular respecto a los demás referente a ruidcs y condiciones generales en que se hallan los barrios residenciales que los rodear.

En efecto; los aeropuertos, particularmente los de origen antiguo, tienen sus pistas de vuelo en zonas exteriores a. los recirtos urbanos de las ciudades que los instalaron. E. tiempo ha ido acercando estas zonas o bien la ciudad îe ensanchando su radio urbano hasta llegar a la situación actual, en la que estos aeropuertos se hallan rodeados de zonas residenciales de considerable importancia.

En estas zonas los ruidos se van desarrollando con molestias crecientes, ya que, por un lado, los motores son más potentes y ruidosos y, por otro, la edificación residencial se va haciendo más densa de año en año.

Con objeto de evitar o atenuar las molestias de estos ruicios se han establecido directrices generales para los acercamientos y direcciones de salida de los aviones, supresión parcial de vuelos nocturncs, entrenamientos, etc. Pero, no obstante esto, la insonorización es cada día más necesaria en estos paraịes.

Los aparatos potentes que calientan motores o que, por cualquier causa han de permanecer en marcha originan grandes ruidos que son difíciles de atenuar, ya que no cabe hablar de evitarlos totalmente. Distintas instituciones de carácter técnico y científico han dirigido sus esfuerzos a solucionar este problema. Una de las conclusiones prácticas que han pasado del orden de ideas a realidad fue el hangar de insonorización, construido recientemente en el aeropuerto de Hamburgo, y de cuya descripción damos un ligero alcance.

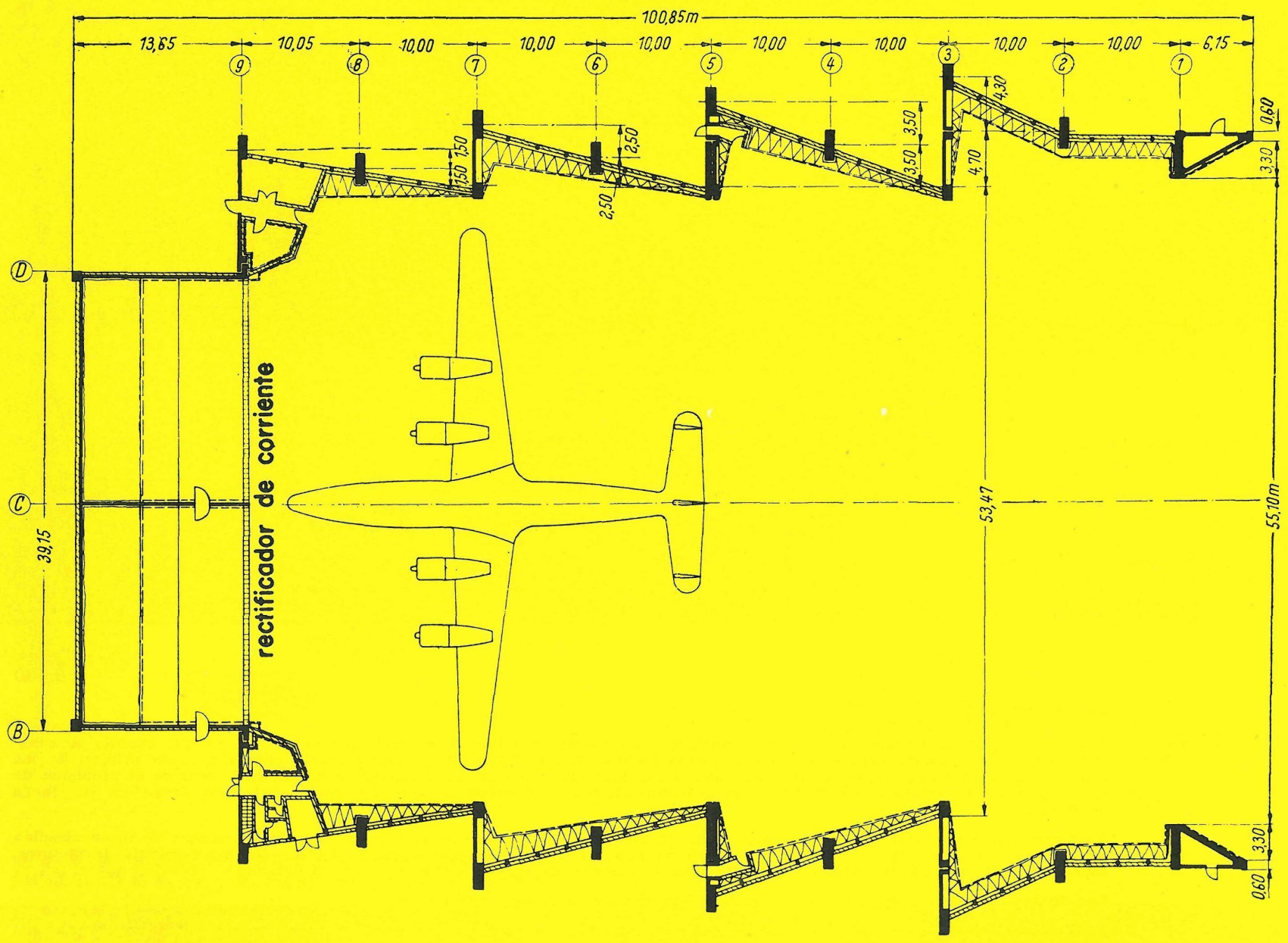

p a 

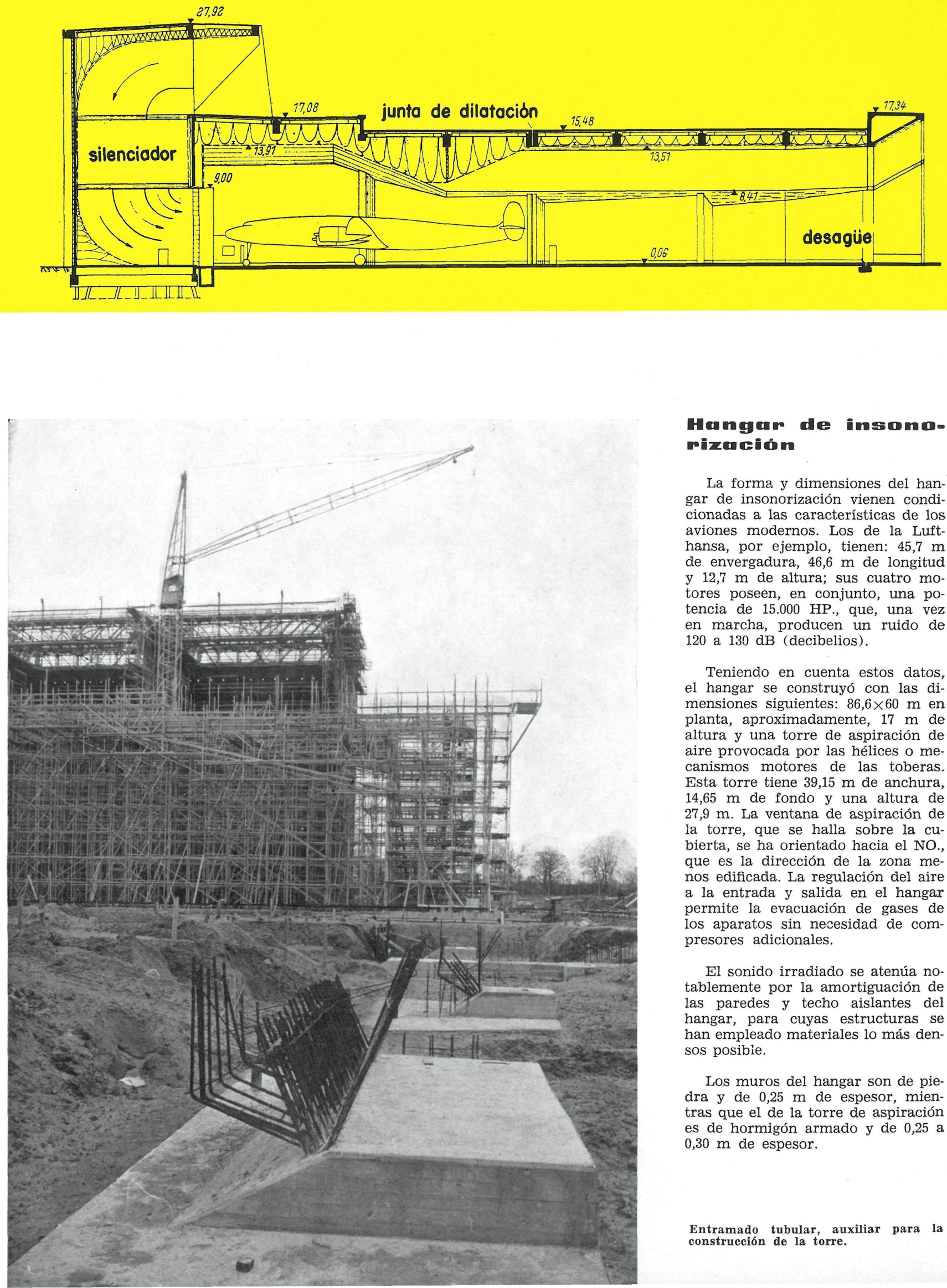

\section{Hangar de insomo- mizctúión}

La forma y dimensiones del hangar de insonorización vienen condicionadas a las características de los aviones modernos. Los de la Lufthansa, por ejemplo, tienen: $45,7 \mathrm{~m}$ de envergadura, $46,6 \mathrm{~m}$ de longitud y $12,7 \mathrm{~m}$ de altura; sus cuatro motores poseen, en conjunto, una potencia de $15.000 \mathrm{HP}$., que, una vez en marcha, producen un ruido de 120 a $130 \mathrm{~dB}$ (decibelios).

Teniendo en cuenta estos datos, el hangar se construyó con las dimensiones siguientes: $86,6 \times 60 \mathrm{~m}$ en planta, aproximadamente, $17 \mathrm{~m}$ de altura y una torre de aspiración de aire provocada por las hélices o mecanismos motores de las toberas. Esta torre tiene $39,15 \mathrm{~m}$ de anchura, $14,65 \mathrm{~m}$ de fondo y una altura de $27,9 \mathrm{~m}$. La ventana de aspiración de la torre, que se halla sobre la cubierta, se ha orientado hacia el NO., que es la dirección de la zona menos edificada. La regulación del aire a la entrada y salida en el hangar permite la evacuación de gases de los aparatos sin necesidad de compresores adicionales.

El sonido irradiado se atenúa notablemente por la amortiguación de las paredes y techo aislantes del hangar, para cuyas estructuras se han empleado materiales lo más densos posibie.

Los muros del hangar son de piedra y de $0,25 \mathrm{~m}$ de espesor, mientras que el de la torre de aspiración es de hormigón armado y de 0,25 a $0,30 \mathrm{~m}$ de espesor.

Entramado tubular, auxiliar para la construcción de la torre. 

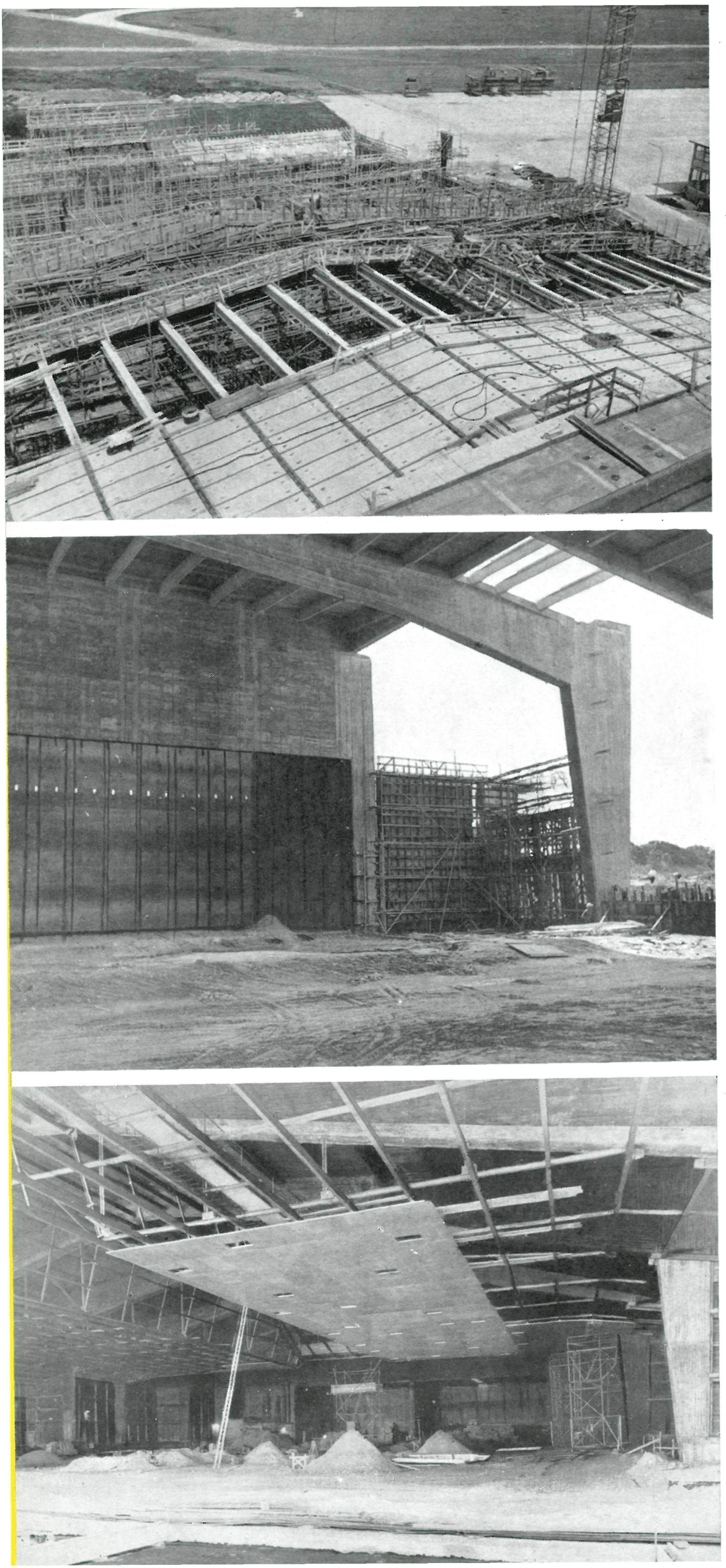

Cerramiento de la cubierta.

Montante de un pórtico.

Techo de insonorización del hangar.
El amortiguamiento del sonido en la nave del hangar se realiza por medio de unos huecos que se han dejado en el techo. Entre estos huecos y la cubierta o paredes laterales existe una zona, también hueca, revestida con esteras y fibras minerales colocadas en forma de zig-zag. Este revesti miento es capaz de absorber hasta un $90 \%$ del sonido procedente del aparato en marcha.

El sonido no absorbido es reflejado hacia el centro del hangar, gracias a la disposición en diente de sierra de los muros laterales del mismo. Esta reflexión aumenta en forma notable el grado de amortiguamiento del hangar. Los ruidos dirigidos hacia la torre de aspiración se reducen por medio de un amortiguador colocado verticalmente en la torre. Este amortiguador está constituido por unos prismas rectangulares, de $8,5 \times 13,2 \times 0,80 \mathrm{~m}$, espaciados a $1,23 \mathrm{~m}$. La torre lleva, además, un revestimiento absorbente y una serie de aspas directrices para desviar el aire aspirado y que se han rellenado con materiales absorbentes del sonido.

La construcción de este hangar ha presentado otros problemas, ya que las hélices requieren un techo de $14 \mathrm{~m}$ de altura y una corriente regular de aire sin torbellinos. Para solventar estas necesidades se ha instalado un rectificador de corriente de $50 \mathrm{~cm}$ de profundidad, células de $25 \mathrm{~cm}$ de altura y $38 \mathrm{~m}$ de anchura; todo esto situado en una extensión de $9 \mathrm{~m}$ de alto y entre la torre de aspiración y la nave del hangar.

\section{Estructurat del thangare}

La estructura del hangar se compone de siete pórticos, de hormigón pretensado, espaciados a $10 \mathrm{~m}$. Cada uno de estos pórticos, con cabezal en forma de $\mathrm{V}$ invertida muy aijierta, vaciado en sus dos caras y pretensado, constituye un elemento de arriostramiento con las correas para absorber los efectos del viento.

La torre de aspiración está formada por muros macizos de hormigón pretensado. 


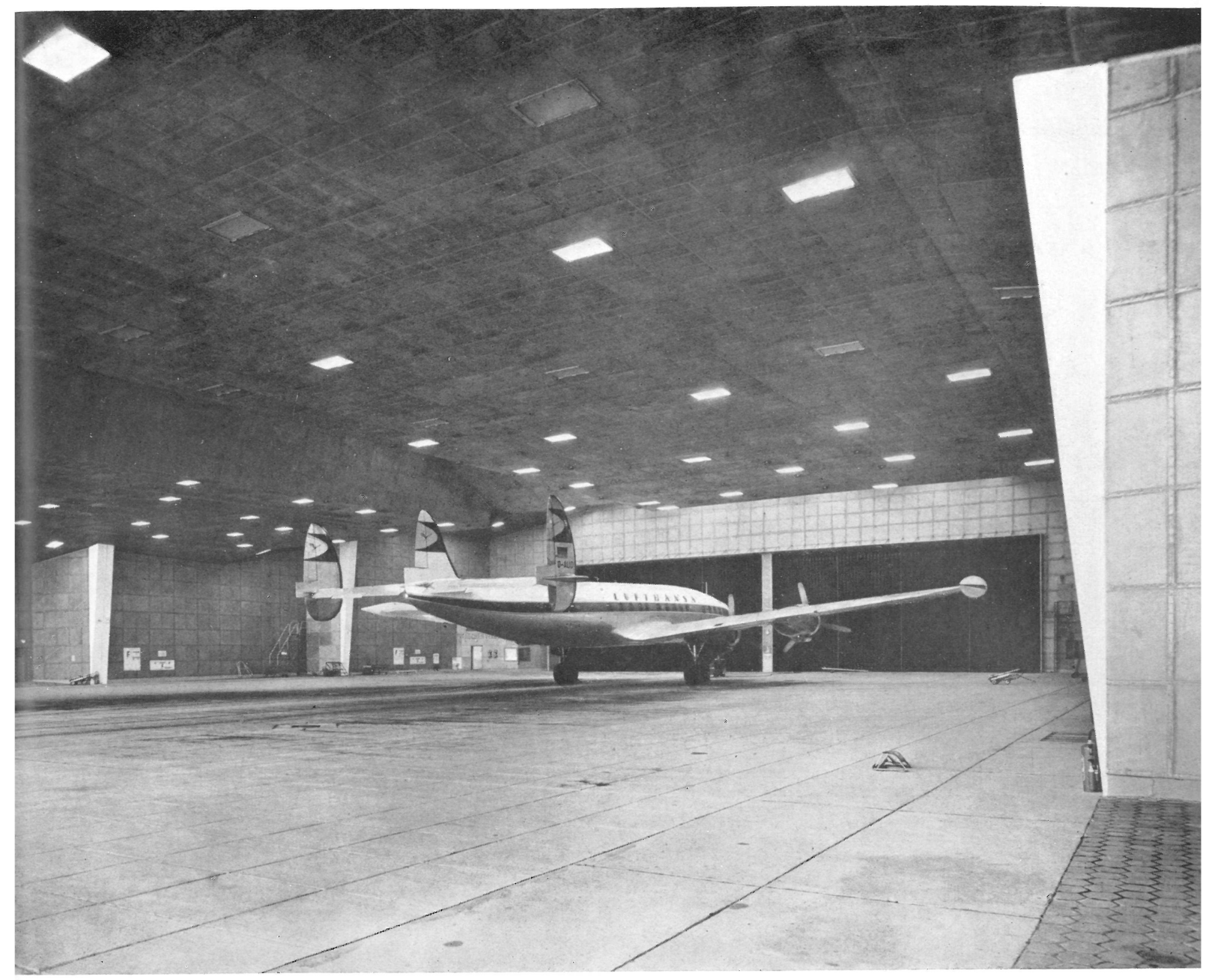

Fotos: BAUBEHÖRDE

Como el terreno no poseía suficiente capacidad de sustentación, fue imprescindible utilizar pilotes de hasta $15 \mathrm{~m}$ cle longitud y $160 \mathrm{t}$ de capacidad cada uno.

Antes de entrar en servicio el hangar, se hicieron diferentes ensayos para comprobar su comportamiento real con los aviones provistos de hélices y sus motores en marcha. Todos estos ensayos se realizaron en presencia de destacadas personalidades de la ingeniería física y construcción, y duraron unas dos semanas.

Los ensayos llevados a cabo con un aparato Constellation, el mayor tipo de aviones de la Lufthansa dieron un nivel sonoro de 60 fonios, que corresponde al ruido que produce un automóvil por la calle y percibido en el interior de una vivienda cerrada. Al aire libre, y con la misma distancia, un avión daría un nivel de 125 fonios. Esta reducción o mo de 1 a $2 / 100$, y no de mitad, como parece ser, puesto que los ruidos no varían linealmente en intensidad. Las reducciones prácticas que con este hangar de insonorización se alcanzan son del orden
de $1 / 5$ del ruido original. Naturalmente, que si se trata de reactores son mayores los ruidos; pero, aún así, los que llegan a las zonas próximas no son nocivos.

La parte estructural del hangar no ha presentado dificultades para su ejecución, que fue desarrollada según los métodos modernos empleados en la construcción mecanizada. 


\section{3.}

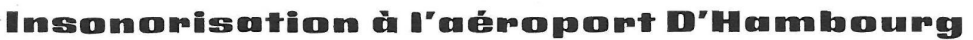

H. Konrad Havemann, Docteur ingénieur.

Les aéroports, notamment les plus anciens, posent actuellement le problème du bruit pour leur situation dans des zones qui, à l'époque où ils furent construits, étaient à peine habitées. L'expansion et l'accroissement continus des grandes villes qui poussent les quartiers résidentiels aux environs des aéroports, posent le problème de l'insonorisation, car on doit éviter, ou tout au moins atténuer, les inconvénients produits par les bruits excessifs dans les zones d'habitation.

Pour trouver une solution à cet état de choses, on a procédé, à Hambourg, à la construction d'un hangar d'insonorisation des bruits que les avions modernes produisent à la mise en marche de leurs moteurs. L'avion passera, à l'intérieur de ce hangar, le temps nécessaire au chauffage de ses moteurs, attente, etc.

Les essais exécutés dans ce hangar en service ont donné des résultats satisfaisants.

Sa structure générale est en béton précontraint avec de forts portiques transversaux. Suspendu au plafond est installé un système de plaques acoustiques destiné à amortir les bruits.

Le hangar est d'une forme originale, puissante et de caractéristiques plastiques.

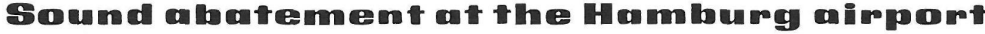

H. Konrad Havemann, Dr. Eng.

Airports, especially the older ones, are faced with the problem of sound intensity. At the time of their construction most of them were situated in sparesely inhabited zones. But the expansion of the urban radius of the large cities has extended the residential areas to the neighbourhood of the airports, and this has given rise to the problem of sound abatement since it is obviously necessary to reduce as far as possible the nuisance which excessive noise creates to the local population.

To overcome one aspect of the problem, the construction of a soundproof hangar has been initiated at Hamburg, so that the noise which the engines make, during their warmingup period, can be controlled. The airliners will be inside this hanger during the period of tuning up, or warming up of the engines.

The tests of this hangar, under working conditions, have proved satisfactory.

The general structure is of prestressed concrete, with strong transversal portal frames. A number of accoustical plates have been supported from the roof, and they will act as sound absorbers.

The exterior aspect of the building is original, showing a powerful design of strong lines.

\section{Lairanschutzhalle des Flughofenss won Hamburg}

H. Konrad Havemann, Dr.-Ingenieur.

Die Flughäfen, besonders die älteren, weisen gegenwärtig das Problem des Geräusches auf, da sie ursprünglich in kaum besiedelten Gebieten angelegt wurden. Die Ausdehnung und das stete Anwachsen des Radius der grossen Städte bringt die Wohngegenden den Flughäfen immer näher, und damit erscheint das Problem des Lärmschutzes. Der übermässige Lärm muss vermieden oder wenigstens abgeschwächt werden, um die Bewohner nicht zu sehr zu belästigen.

Um diesem Tatbestand eine Abhilfe zu schaffen, hat man es in Hamburg unternommen, eine Schutzhalle gegen den Lärm, den die modernen Maschinen beim Laufen ihrer Motoren verursachen, zu bauen. Die Zeit des Erhitzens der Motoren, des Wartens, u.s.w. verbringen die Maschinen im Innern dieser Halle.

Die an der sich im Betrieb befindenden Halle durchgeführten Prüfungen haben zufriedenstellende Ergebnisse gezeitigt.

Das allgemeine Tragwerk ist aus Spannbeton mit starken Querrahmen. Am Dache und an den Wänden wurde ein System schalldämpfender Platten angebracht, welches zum Schlucken des Lärmes dient.

กэร Gebäude stellt eine originelle, starke Form von bezeichnender Plastik dar. 